Incident infections and PID diagnoses were determined in women who attended clinic more than once and did not have PID at enrolment. Crude incidence rates of PID were calculated. Cox proportional hazards regression was used to investigate the association between chlamydia, gonorrhoea and PID.

Results 275 women with 387 person years (py) of follow-up were diagnosed with 85 cases of chlamydia, 34 cases of gonorrhoea, 25 cases of trichomoniasis, 253 cases of BV and 148 cases of candida. Thirty-eight women had at least one episode of PID and of these, 24 had chlamydia concurrently or preceding their PID. Nine women presented with both chlamydia and PID, 1 had concurrent gonorrhoea and PID, and 3 had gonorrhoea during the 8 weeks preceding their PID. The crude incidence rate was 16.03 cases per 100 py $(95 \%$ CI 12.50 to 20.56) for chlamydia and 6.72 cases per 100 py (95\% CI 4.58 to 9.87 ) for gonorrhoea. The crude rate and crude and adjusted HRs of PID were calculated, with women censored after their first episode of PID, for all women and for women classified according to their exposure to chlamydia and gonorrhoea (Abstract P1-S3.09 table 1).

Conclusions In this cohort chlamydia was a risk factor for PID. After controlling for other factors, exposure to chlamydia at enrolment was a borderline predictor of PID while incident infection was not associated, possibly due to the small number of incident cases or to the reduced risk because of prompt treatment. The absolute risk of PID after an incident case was higher than recent estimates which may be due to the greater sensitivity of current chlamydia tests. This emphasises the importance of using contemporary parameters when modelling the cost-effectiveness of chlamydia screening.

\section{P1-S3.10 THE ROLE OF CHLAMYDIA TRACHOMATIS IN THE DEVELOPMENT OF SYMPTOMATIC PELVIC INFLAMMATORY DISEASE: A MULTI-PARAMETER SYNTHESIS}

doi:10.1136/sextrans-2011-050108.143

${ }^{1} \mathrm{M}$ Price, ${ }^{1} \mathrm{~T}$ Ades, ${ }^{1} \mathrm{~N}$ Welton, ${ }^{1} \mathrm{~J}$ Macleod, ${ }^{2} \mathrm{~K}$ Soldan, ${ }^{3} \mathrm{D}$ De Angelis, ${ }^{2} \mathrm{~S}$ Simms, ${ }^{1} \mathrm{~K}$ Turner, ${ }^{1} \mathrm{P}$ Horner. ${ }^{1}$ University of Bristol, Bristol, UK; ${ }^{2}$ Health Protection Agency, UK; ${ }^{3}$ Medical Research Council Biostatistics Unit, UK

Background Chlamydia trachomatis (CT) is an important risk factor for the development of Pelvic Inflammatory Disease (PID), but despite much study the extent of its causal role remains unclear. Knowledge of the role of Chlamydia is critical for assessing the costeffectiveness of screening programmes.

Methods We perform a Bayesian multi-parameter synthesis of evidence from a range of prospective, retrospective, and routine data sources. These were; randomised trials following screened and unscreened women to PID; retrospective studies of Chlamydia in PID cases and controls, from which an etiologic fraction can be estimated; routine data on the number of PID cases diagnosed in England in 1 year; survey data on the probability of diagnosis of PID. We also used information on the incidence and prevalence of CT in England and the clearance rate of asymptomatic infection.

Results We found that the different data sources provided consistent estimates of the critical parameters. We estimate the probability an episode of Chlamydia causes an episode of PID to be 0.09 (0.04 to 0.16 ) and that the Excess fraction of PID cases due to Chlamydia to be 0.20 (0.09 to 0.35$)$. We estimate that prior to the start of the current screening program in the UK the annual population incidence of PID in women in England was 0.022 (0.016 to 0.028) in women aged $16-24$ and $0.013(0.009$ to 0.019$)$ in women aged $25-44$ and that $42 \%$ (31\% to $54 \%$ ) of these cases were diagnosed.
Conclusions In the absence of direct data, it is possible to statistically combine evidence from different types of data, to check the consistency of the data sources, and to estimate the relation between CT and PID. In considering the effect on PID risk of treating women who screen positive for Chlamydia, it is important to note that this is a survivor population who acquired CT an unknown time previously and who have not developed PID.

\section{Epidemiology poster session 3: Burden of disease: Neonatal herpes
P1-S3.11 FACTORS ASSOCIATED WITH DEATH AMONG INFANTS WITH NEONATAL HERPES REPORTED IN NEW YORK CITY, $2006-2010$

doi:10.1136/sextrans-2011-050108.144

${ }^{1,2} \mathrm{~J}$ Schillinger, ${ }^{1} \mathrm{~S}$ Handel, ${ }^{1} \mathrm{~K}$ Washburn, ${ }^{1} \mathrm{E}$ Klingler, ${ }^{1,2} \mathrm{~S}$ Blank, ${ }^{1} \mathrm{P}$ Pathela. ${ }^{1} \mathrm{~N}$ New York City Department of Health, New York, USA; ${ }^{2}$ US Centers for Disease Control, New York, USA

Background Neonatal herpes simplex virus infection (nHSV) has a high case fatality rate. In New York City (NYC), healthcare providers (HCP) and laboratories report nHSV to the Health Department; incidence is highest among infants born to black nonHispanic (NH), and young women. We analysed nHSV cases to identify factors associated with death.

Methods nHSV cases in infants $<60$ days were investigated by HCP interview and abstraction of labour and delivery, outpatient, inpatient, vital records. Bivariate and multivariate models estimated associations between death and demographic, clinical, and provider factors.

Results During 2006-2010, 71 laboratory-confirmed nHSV cases were reported; 43/71 (55\%) were male, 29/71 (41\%) HSV type 1, 28/ 71 (39\%) HSV type 2, and 14/71 (20\%) untyped. Among 58 cases with recorded maternal race/ethnicity, 19 (33\%) were white $\mathrm{NH}, 25$ $(43 \%)$ were black NH, and $15(26 \%)$ were Hispanic. Fourteen of the $71(20 \%)$ cases died; 10 of the deaths $(71 \%)$ were among infants born to black $\mathrm{NH}$ women. There was a significant association between death and disseminated disease (vs skin/eye/mucous membrane, central nervous system, or congenital infection) (OR 25.8, 95\% CI 4.5 to 148.9), black NH maternal race (OR 10.0, 95\% CI 2.4 to 41.0 ), preterm birth (OR 7.1, 95\% CI 1.9 to 26.5), age $<7$ days at first symptom (OR 5.8, 95\% CI 1.5 to 23.3 ), maternal age $<26$ (OR 3.02, 95\% CI 0.8 to 10.86), vaginal delivery (OR $0.2,95 \%$ CI 0.1 to 0.8 ), and presence of infant herpes lesions (OR $0.2,95 \% \mathrm{CI}$ 0.04 to 0.6 ). Infant sex, fever, viral type, admitting hospital type (academic vs non-academic), illness within $24 \mathrm{~h}$ of birth, and duration rupture of membranes were not associated with death. After adjusting for preterm birth, black $\mathrm{NH}$ maternal race remained associated with a significantly higher risk for death (OR 7.2 95\% CI 1.6-to 31.4, $\mathrm{p}=0.009)$. Disseminated disease also remained significantly associated with death after adjusting for preterm birth, and age at first symptom (OR 26.6, 95\% CI 3.6 to 196.5, $\mathrm{p}=0.001$ ).

Conclusions Our data suggest a significant racial disparity in nHSV fatality, only partly explained by preterm births among black $\mathrm{NH}$ mothers. Further investigation should examine if care-seeking or provider characteristics contribute to these disparities. Black $\mathrm{NH}$ maternal race may be a marker for recently acquired herpes infection, with a high organism load at delivery. Disseminated disease is strongly, and independently, associated with fatality. 